Brian Ash, MD

San Francisco, CA, USA

Eric Widra, MD

Washington, DC, USA

\section{A New Simulation Model for Skin Abscess Identification and Management}

Objective.-Cutaneous abscesses are common, and abscess identification with incision and drainage is a curative procedure that can be performed in out-of-hospital environments. While medical simulation models can enable proficiency in such skills, current abscess models described in the literature suffer from limitations. The author presents a novel abscess management training simulator evaluated by physicians.

Methods.-To create the simulator, mock purulent material is injected into an artificial "abscess wall" that has been tunneled near the surface of a chicken breast. The simulation model was evaluated by 20 physicians familiar with abscess identification and management. Evaluators assessed the model and answered 2 closed-ended questions regarding the model with an opportunity for open-ended feedback. Physicians were then shown ultrasound images of the simulated abscess along with 2 real abscesses and evaluated the sonographic fidelity of the ultrasound model via a closed-ended question.

Results.-All 20 physician evaluators agreed that an abscess simulator model would be a useful teaching tool and that this particular abscess model would be a useful teaching tool. Evaluators commented on the excellent realism of the model and also a limitation from an absence of mock purulent loculations. When evaluators responded to the statement "the ultrasound image of the simulated abscess appears realistic," $10 \%$ of individuals "strongly agreed" and 90\% of individuals "agreed" with this statement $(\mathrm{n}=20)$.

Conclusions.-This new simulation model is realistic, simple to create, and may be an effective tool to teach cutaneous abscess management. Physicians who evaluated the simulated abscess found that it replicates the classic palpable fluctuance and ultrasound findings of an actual abscess and can be surgically incised and drained in a similar fashion. While physicians agreed that this model would be useful, future studies may validate this task trainer as an effective teaching tool.

Jason D. Heiner, MD

Tacoma, WA, USA

\section{0\% Povidone-Iodine May Be a Practical Field Water Disinfectant}

Objective.-The search for an ideal field water disinfectant persists, despite many available products. While other forms of iodine have demonstrated effectiveness, there is a paucity of data available regarding the use of iodophores such as povidone-iodine (PVI) to disinfect water. Escherichia coli is the most common cause of traveler's diarrhea and is found in tropical, temperate, and cold waters. We sought to experimentally determine the minimal disinfecting concentration of $10 \%$
PVI over different contact times when added to water inoculated with $E$ coli at varying water temperatures.

Methods.-Full-strength 10\% PVI was added to sterile deionized water to create 1:100,1:1000, and 1:10 000 dilutions. E coli were exposed to these strengths of PVI for 5, 15, and 30 minutes. The bactericidal activity of PVI was neutralized with $0.5 \%$ sodium thiosulfate. The number of viable colony forming units was determined after 24 hours of incubation at $37^{\circ} \mathrm{C}$ on Luria-Bertani agar. Effective bactericidal activity was defined as a $5-\log$ reduction $(99.999 \%$ bacterial kill). This procedure was repeated at $10^{\circ} \mathrm{C}, 20^{\circ} \mathrm{C}$, and $30^{\circ} \mathrm{C}$.

Results.-The lowest concentration of 10\% PVI resulting in effective disinfection of water inoculated with $E$ coli was the 1:1000 dilution and was seen after 5 minutes of contact time. The 1:100 concentration of 10\% PVI always resulted in effective disinfection, while the 1:10 000 concentration never effectively disinfected the water. No significant differences were observed across the experimental temperatures.

Conclusions.-A 10\% PVI concentration between 1:1000 to 1:10 000 has been suggested by past authors as a possible but unproven strength for water disinfection. Our experiment supported this suggestion against $E$ coli, the organism most commonly responsible for traveler's diarrhea. This amount of available iodine per liter of treated water is safe, but further studies are needed to assess the effectiveness of PVI against more virulent waterborne pathogens.

Jason D. Heiner, MD

David Hile, MD

Samandra T. Demons, $\mathrm{PhD}$

Ian Wedmore, MD

Tacoma, WA, USA

\section{The Practicality of Backcountry Water Purification Units for Wound Irrigation}

Background.-Each year millions of Americans venture to the backcountry to enjoy the outdoors. Lacerations and abrasions are some of the most common injuries encountered. Previous research shows that the best treatment to limit infection in contaminated wounds is irrigation of the wound with pressures between 6 and 15 pounds per square inch (psi).

Methods.-We took 4 of the most popular water purifications units available to the consumer and measured the impact pressures of each to see if they would consistently produce the required $6 \mathrm{psi}$ necessary for adequate wound irrigation. Impact pressures were calculated by directing the stream onto a metal bending beam that allowed measurement of force. PSI was then calculated by dividing the force of the stream by its surface area.

Results.-Using 3 separate measurements added together and divided for 1 average the following psi were calculated. Katadyn Hiker Pro, 1.38 psi; MSR Sweetwater microfilter, 3.68 psi; First need deluxe water purifier, $1.84 \mathrm{psi}$; and MSR Miniworks EX microfilter, 0.82 psi.

Conclusions. - While any type of irrigation and cleansing of a contaminated wound is better than nothing, none of the 4 water purification units reached the suggested 6 psi necessary for wound irrigation. It is suggested that a $35-$ to $50-\mathrm{mL}$ syringe 